\title{
The Velocity Field from Type la Supernovae Matches the Gravity Field from Galaxy Surveys
}

\section{Citation}

Riess, Adam G., Marc Davis, Jonathan Baker, and Robert P. Kirshner. 1997. "The Velocity Field from Type la Supernovae Matches the Gravity Field from Galaxy Surveys." The Astrophysical Journal 488 (1): L1-5. https://doi.org/10.1086/310917.

\section{Permanent link}

http://nrs.harvard.edu/urn-3:HUL.InstRepos:41399823

\section{Terms of Use}

This article was downloaded from Harvard University's DASH repository, and is made available under the terms and conditions applicable to Other Posted Material, as set forth at http:// nrs.harvard.edu/urn-3:HUL.InstRepos:dash.current.terms-of-use\#LAA

\section{Share Your Story}

The Harvard community has made this article openly available.

Please share how this access benefits you. Submit a story.

Accessibility 


\title{
THE VELOCITY FIELD FROM TYPE IA SUPERNOVAE MATCHES THE GRAVITY FIELD FROM GALAXY SURVEYS
}

\author{
Adam G. Riess, ${ }^{1}$ Marc Davis, ${ }^{1}$ Jonathan Baker, ${ }^{1}$ And Robert P. KirshneR ${ }^{2}$ \\ Received 1997 June 25; accepted 1997 July 29; published 1997 September 9
}

\begin{abstract}
We compare the peculiar velocities of nearby Type Ia supernovae (SNs) with those predicted by the gravity fields of full-sky galaxy catalogs. The method provides a powerful test of the gravitational instability paradigm and strong constraints on the density parameter $\beta \equiv \Omega^{0.6} / b$. For 24 Type Ia SNs within $10,000 \mathrm{~km} \mathrm{~s}^{-1}$, we find that the observed Type Ia SN peculiar velocities are well modeled by the predictions derived from the $1.2 \mathrm{Jy}$ IRAS survey and the Optical Redshift Survey (ORS). Our best $\beta$ value is 0.4 from IRAS, and 0.3 from the ORS, with $\beta>0.7$ and $\beta<0.15$ ruled out at $95 \%$ confidence levels from the IRAS comparison. Bootstrap resampling tests show these results to be robust in the mean and in its error. The precision of this technique will improve as additional nearby Type Ia SNs are discovered and monitored.
\end{abstract}

Subject headings: cosmology: observations — large-scale structure of universe — Local Group supernovae: general

\section{INTRODUCTION}

The motions of galaxies probe the size of potentials formed by the gravitating matter in the universe. Redshift surveys map the spatial distribution of luminous matter. Together, the two measure the contribution of luminous and dark matter to the contents of the universe, providing a direct measure of the density parameter on the largest possible scale.

Previous attempts to test the gravitational instability paradigm and constrain the mass density parameter, $\Omega_{0}$, are hindered by imprecise distance estimates to individual galaxies. Distance indicators based on empirical relationships between galaxy luminosity and internal velocity (i.e., Tully-Fisher and $D_{n}-\sigma$ ) yield individual distance uncertainties of 20\%-25\% (Jacoby et al. 1992; Tully \& Fisher 1977; Dressler et al. 1987; Willick et al. 1996). To increase the utility of such data, workers have amassed it in great quantity. While this strategy diminishes the random component of error, imprecise distances give rise to more troublesome systematic errors, including selection bias, Malmquist bias, and smoothing biases (Strauss \& Willick 1995). The antidote for such biases depends on knowledge of the distance uncertainty, a controversial quantity (Willick et al. 1995; Mathewson \& Ford 1994; Federspiel, Sandage, \& Tammann 1994). The forced marriage of inhomogeneous catalogs could result in additional errors in the inferred velocity field. Despite these challenges, remarkable progress has been made in this field (Dekel 1994; Dekel, Bertschinger, \& Faber 1990; Davis, Nusser, \& Willick 1996; Hudson 1994; Shaya, Peebles, \& Tully 1995; Nusser \& Davis 1994).

Type Ia supernovae (SNs) are well suited to provide an independent test of the gravitational instability paradigm and to constrain the mass density. Light curves from the Calán/Tololo Survey (Hamuy et al. 1993, 1996) and the CfA survey (Riess 1996; Riess et al. 1997) yield distances with 5\%-10\% uncertainty over the redshift range $1000 \leq c z \leq 36,000 \mathrm{~km} \mathrm{~s}^{-1}$. Although the sample of observed Type Ia SNs is relatively small, the depth and precision of SN distances provide some advantages. For the reduction of random errors, one Type Ia SN is

\footnotetext{
${ }^{1}$ Department of Astronomy, University of California, Berkeley, CA 947203411.

${ }^{2}$ Harvard-Smithsonian Center for Astrophysics, 60 Garden Street, Cambridge, MA 02138 .
}

worth $\sim 10$ Tully-Fisher or $D_{n}-\sigma$ measurements. Systematic bias, which rises with the square of the distance uncertainty, is also $\sim 10$ times smaller for Type Ia SN distances. Individual distance errors can be derived from Type Ia SN light curves (Riess, Press, \& Kirshner 1995a, 1996), which give meaningful measurements of the deviation from smooth Hubble flow. Combining the Type Ia SN data with models of the predicted "peculiar velocities," one can constrain the mass density traced by galaxy fluctuations while testing the gravitational instability model for structure formation.

\section{ANALYSIS}

The direct comparison of observed peculiar velocities and mass density fluctuations is not trivial. The problem becomes tractable only by assuming that the large-scale component of the flow is single valued and irrotational. Since galaxy fluctuations on scales larger than $10 h^{-1} \mathrm{Mpc}$ are observed to be less than unity (Davis \& Peebles 1983), these assumptions are reasonable. Furthermore, for such fluctuations at late times, linear perturbation theory is quite accurate, and the expected velocity field $\boldsymbol{v}_{p}$ can be related to the peculiar gravity field $\boldsymbol{g}$ (Peebles 1980) by

$$
\boldsymbol{v}_{p}(r)=\left(\frac{2}{3 \Omega_{0}^{0.4} H_{0}}\right) \boldsymbol{g}(\boldsymbol{r}) .
$$

This equation simply states that the observed peculiar velocity results from the gravitational acceleration acting over a Hubble time.

The gravity field $\boldsymbol{g}(\boldsymbol{r})$ may be inferred from the distribution of galaxies that are assumed to trace the matter field. It is common to assume that an unknown but linear bias exists between galaxy fluctuations $\delta_{G}$ and matter fluctuations $\delta_{\rho}$ by employing a bias parameter $b$, i.e., $\delta_{G}=b \delta_{\rho}$. This simplification, while not accurate on small scales or in dense environments, should suffice on large scales.

Using linear theory, one can constrain only a combination of $\Omega_{0}$ and $b, \beta \equiv \Omega_{0}^{0.6} / b$. A variety of methods for estimating $\boldsymbol{g}(\boldsymbol{r})$ from galaxy surveys are reviewed by Strauss \& Willick (1995). Here, we use the method of Nusser \& Davis (1994), which can successfully repair the gravitational distortions in 


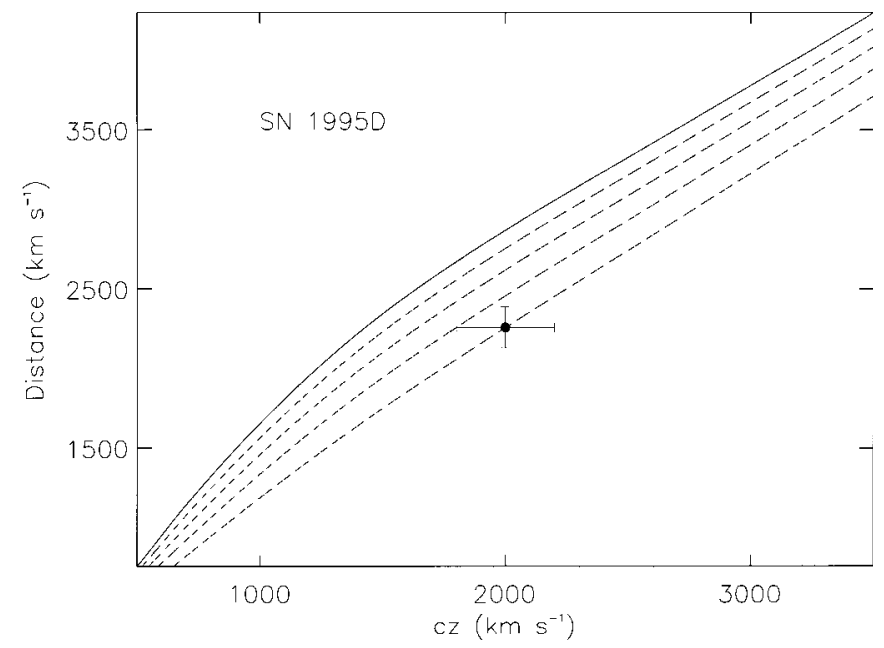

FIG. 1.-Velocity-distance relation in the vicinity of Type Ia SNs. This is an example of the velocity vs. distance predictions of the IRAS gravity maps as a function of the mass density parameter, $\beta$, in the direction of Type Ia SNs. The solid line shows the $\beta=1.0$ prediction, with subsequent dotted lines showing the predictions for $\beta$ in decreasing increments of 0.2 . Overplotted is the measurement of the Type Ia SN redshift, MLCS distance (in $\mathrm{km} \mathrm{s}^{-1}$ ), and distance error with a small-scale velocity error of $200 \mathrm{~km} \mathrm{~s}^{-1}$. Such plots contribute to the determination of $\beta$ via eq. (2).

redshift space. However, this technique cannot properly treat regions with multivalued relations between velocity and distance, expected near clusters of galaxies. The minimum smoothing scale of the derived gravity field is $500 \mathrm{~km} \mathrm{~s}^{-1}$, and this smoothing increases with distance in proportion to the mean interparticle spacing of the galaxy catalog. This increase is necessary to suppress artificial two-body acceleration (Strauss et al. 1992).

For comparison to the Type Ia SN peculiar velocities, we computed the gravity field from the 1.2 Jy flux limited IRAS redshift survey of galaxies (Fisher et al. 1995). This catalog contains 6010 galaxies with median redshift of $6000 \mathrm{~km} \mathrm{~s}^{-1}$ and provides a useful measure of the density field out to $c z \approx 15,000 \mathrm{~km} \mathrm{~s}^{-1}$. The IRAS catalog is known to underrepresent the elliptical-rich cores of clusters relative to optical catalogs. Thus, it is of interest to use the Optical Redshift Survey (ORS) catalog of optically selected galaxies for an alternative calculation of the gravity field (Santiago et al. 1995; Baker et al. 1997). The ORS contains 8457 galaxies, supplemented by the 1.2 Jy IRAS survey in sky regions without optical coverage.

We measured the distances and their uncertainties for 25 Type Ia SNs within $10,000 \mathrm{~km} \mathrm{~s}^{-1}$ using multicolor light-curve shape (MLCS) distance measurements (Riess et al. 1996). The redshifts of the Type Ia SNs come from Hamuy et al. (1996), Riess (1996), and Riess et al. (1997). Because of the limitation of linear biasing and the risk of multivalued flows, we conservatively discarded one Type Ia SN, SN 1992G, because of its proximity to the Virgo Cluster. In the directions of the remaining SNs, we computed the expected distance-redshift relations, based on the gravity fields of either the ORS or IRAS surveys, as a function of $\beta$. Figure 1 shows an example of these curves. We assume a redshift error of $200 \mathrm{~km} \mathrm{~s}^{-1}$ as an estimate of the small-scale component of the radial peculiar velocity not describable by linear theory.

For each SN, we have the distance $d_{i}$ (in $\mathrm{km} \mathrm{s}^{-1}$ ), a distance error $\delta d_{i}$ (in $\mathrm{km} \mathrm{s}^{-1}$ ), a redshift $z_{i}$, and a "redshift error,"

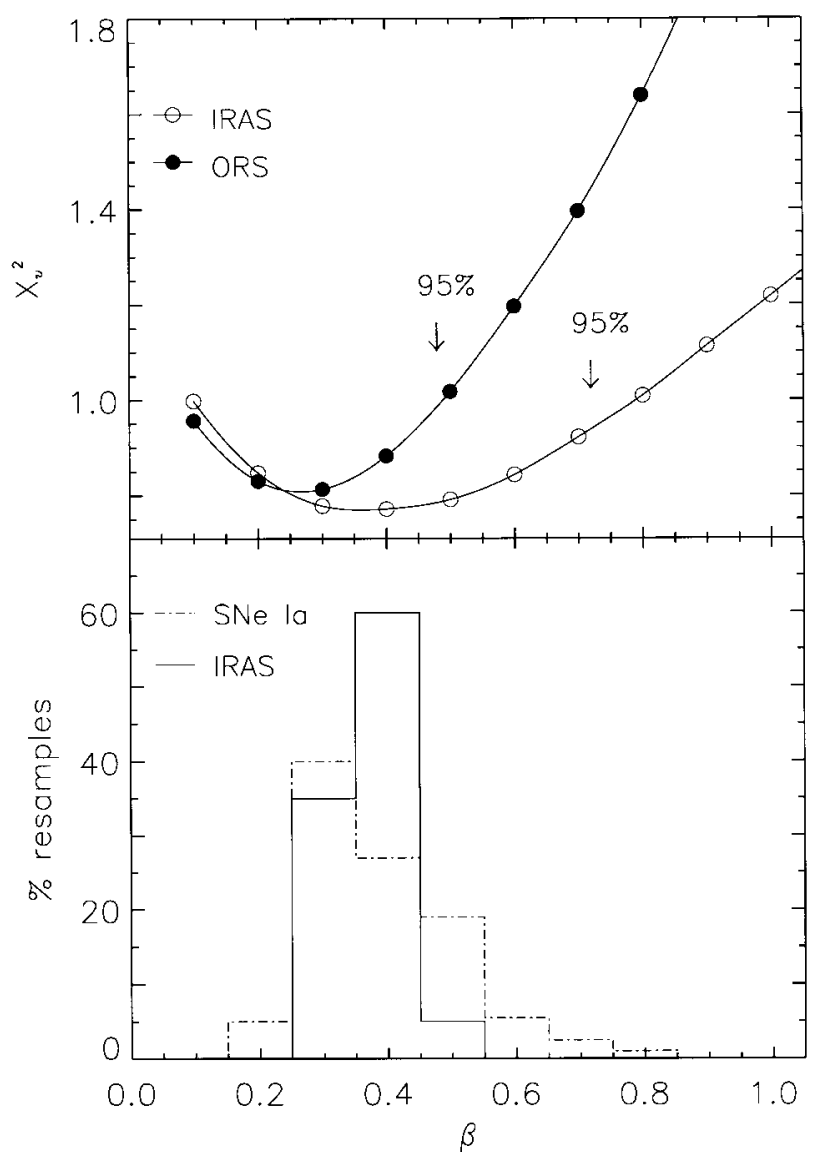

FIG. 2.-Constraints on the mass density parameter, $\beta$, and tests of robustness. (Top) The value of our $\chi^{2}$ statistic as a function of $\beta$ (eq. [3]) reduced (divided) by the 23 degrees of freedom. Our best $\beta$ value is 0.4 from IRAS, and 0.3 from the ORS, with $\beta>0.7$ and $\beta<0.15$ ruled out at $95 \%$ confidence levels for the comparison to IRAS. Bootstrap resamplings (bottom) of the Type Ia SNs and IRAS galaxies, described in $\S 3$, validate our estimates of $\beta$.

$\delta c z_{i}=200 \mathrm{~km} \mathrm{~s}^{-1}$. From the gravity maps, we have the functions $z(j, \beta)$ and $d(j, \beta)$ along a set of points $j$ toward the direction of each SN. We seek the minimum separation of each point from the predicted curves, in units of the standard deviations. That is, for the $i$ th SN Ia, we compute a contribution to a $\chi^{2}$,

$$
\delta \chi(\beta)_{i}^{2}=\min _{j}\left\{\left[\frac{d_{i}-d(j, \beta)}{\delta d_{i}}\right]^{2}+\left[\frac{z_{i}-z(j, \beta)}{\delta z_{i}}\right]^{2}\right\},
$$

where the minimization is over the locus of points $j$ that defines the curve $z(d, \beta)$ toward each SN. A goodness of fit is computed by summing over all the SNs,

$$
\chi(\beta)^{2}=\sum_{i} \delta \chi(\beta)_{i}^{2},
$$

with results shown in Figure 2 (top) for both the IRAS and ORS surveys.

For the IRAS survey, we find $\beta_{I R A S}=0.40 \pm 0.15$, and for the ORS survey we find $\beta_{\mathrm{ORS}}=0.30 \pm 0.10$. In both cases the value of $\chi^{2}$ at the minimum is within the expected tolerance, confirming gravitational instability as a valid model for the observed peculiar motions of Type Ia SNs. We find 
$\left(\beta_{\mathrm{ORS}}\right) / \beta_{\text {IRAS }}=0.75 \pm 0.38$, in good agreement with the relative biasing of 0.7 derived from the correlation functions of ORS and IRAS galaxies (Fisher et al. 1994).

The predicted and observed peculiar velocity fields for the best values of $\beta$ are shown in Figure 3 in the Local Group (LG) frame at the location of each of the 24 Type Ia SNs; these numbers are also listed in Table 1. Although the gravity predictions are derived independently from the observed peculiar velocities, the similarity of the two is remarkable. The leverage any single Type Ia SN measurement has in determining $\beta$ depends on its location. Because the dominant feature of the flow is dipolar, Type Ia SNs along the axis of this dipole carry more weight than those whose radial motion is perpendicular to it. Although the SN data can be extended to greater depth, beyond $10,000 \mathrm{~km} \mathrm{~s}^{-1}$, the sampling of the IRAS and optical surveys is inadequate to derive useful peculiar velocity predictions.

\section{TESTS OF ROBUSTNESS}

To verify that our results are free from the vagaries of our SN and galaxy samples, we performed bootstrap resampling tests on both data sets. This procedure tests the effects our choice of sample points and their uncertainties have on the estimates of $\beta$ by drawing new samples of data from our best estimate of the underlying population: our sample (Press et al. 1992).

We first drew 200 sets of randomly chosen Type Ia SNs from the sample of 24 Type Ia SNs, drawing each object with a Poisson probability of expectation value of 1 . We then subjected each set to our maximum likelihood estimator for $\beta$, using the gravity field of the IRAS catalog. As seen in Figure 2 (bottom), the distribution for $\beta$ preferred by the individual Type Ia SN agrees well with the single likelihood distribution estimated from our sample.

Similarly, we tested the robustness of the IRAS gravity field by generating 20 bootstrap resampled IRAS catalogs, as we did for the resampled SNs catalogs. For each resampled IRAS catalog, we generated full gravity fields for the range $0.1 \leq \beta \leq$ 1.2. The resulting $\beta$ values derived from the minimum $\chi^{2}$ value of the $24 \mathrm{SN}$ sample are also shown in Figure 2 (bottom). Again, this distribution is consistent with the constraints for $\beta$ inferred from the $\chi^{2}$ value of the original data sets. Thus, we believe the estimates in $\S 2$ are reasonable.

\section{DISCUSSION}

Figure 3 demonstrates the remarkable consistency between the observed peculiar velocities of 24 Type Ia SNs and those predicted from the gravity fields of optical or IRAS galaxies using linear perturbation theory and the best value for $\beta$. The excellent $\chi^{2}$ fits in Figure 2 confirm our simple model for the source of peculiar velocities while putting useful constraints on the mass density parameter, $\beta$.

The signals, seen in Figure 3 , are largely dipole patterns revealing the motion of the $\mathrm{LG}$ relative to the $\mathrm{SN}$ frame. They constrain the shear in the large-scale velocity field induced by the gravity of galaxies within the sample's $10,000 \mathrm{~km} \mathrm{~s}^{-1}$ radius. The bulk of the cosmic microwave background dipole signature does appear to have been generated within this radius. Velocity dipole patterns that match the gravitational dipole signature have been detected in several other surveys (e.g., Riess, Press, \& Kirshner 1995b; da Costa et al. 1996; Giovanelli et al. 1996), but flows inconsistent with the predicted gravity field for all $\beta$ values have also been reported (Lauer \& Postman 1992, 1994; Davis et al. 1996).

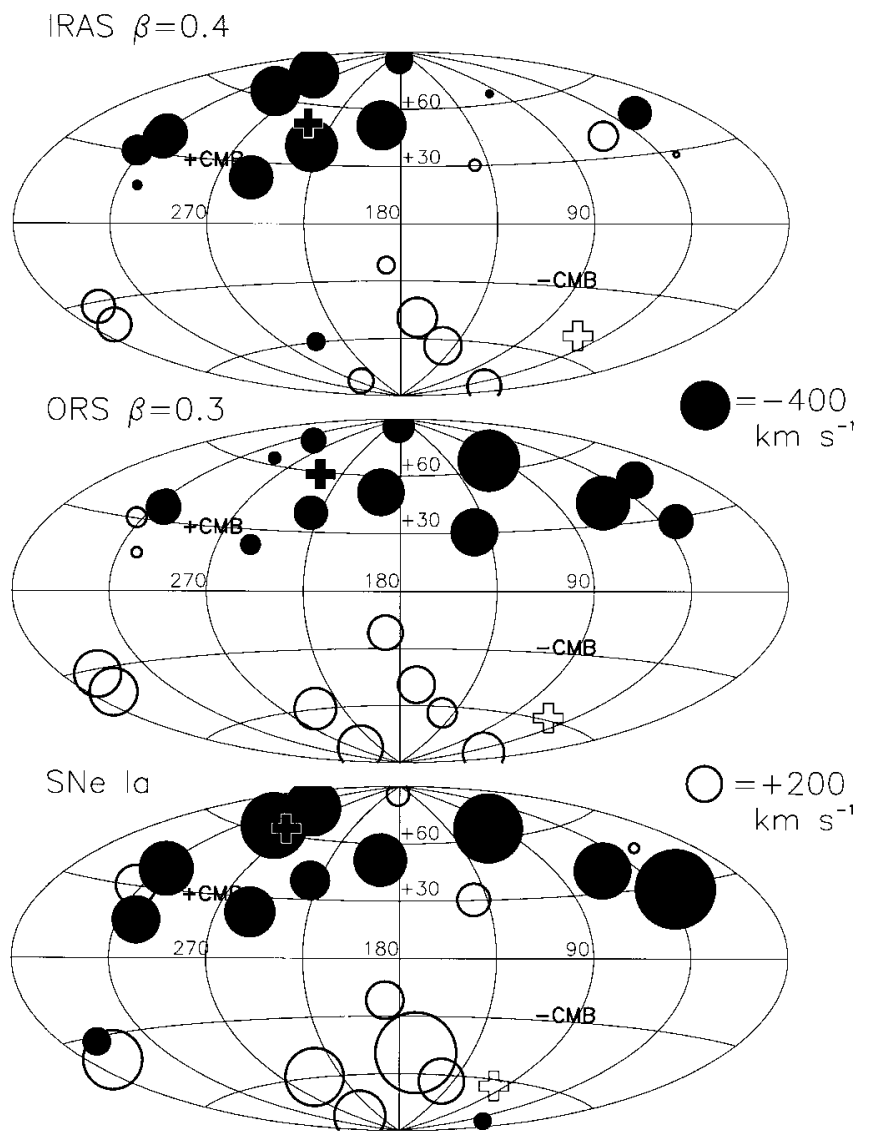

FIG. 3.-Predicted and observed peculiar velocity fields in the Local Group rest frame. Filled/open circles represent Type Ia SNs with measured negative/ positive peculiar velocities (bottom map). The top two maps show the peculiar velocities predicted by the gravity fields of the IRAS and ORS catalogs at the position of the Type Ia SNs for values of $\beta$ that best fit the Type Ia SN velocity field. Filled/open crosses mark the direction toward which the Local Group is approaching/receding. The directions of increased/decreased cosmic microwave background temperature are indicated by $+\mathrm{CMB} /-\mathrm{CMB}$.

A more sophisticated, normal mode comparison of the IRAS gravity field to the velocity field derived from a sample of 2900 Mark III Tully-Fisher galaxies within a limiting redshift of 6000 $\mathrm{km} \mathrm{s}^{-1}$ shows inconsistencies for any value of $\beta$ (Davis et al. 1996). This same procedure applied to the SFI catalog yields consistent gravity and velocity fields with $\beta_{I R A S}=0.6 \pm 0.1$ (da Costa et al. 1997). The POTENT reconstruction of the local density field from the Mark III catalog recovers many observed features in the IRAS maps and estimates $\beta=0.89 \pm 0.12$, but some inconsistencies persist (Sigad et al. 1997; Dekel 1994). The failure to match the fields remains unexplained.

By limiting the analysis to a redshift of $3000 \mathrm{~km} \mathrm{~s}^{-1}$, Willick et al. (1996) successfully applied the VELMOD algorithm to compare the IRAS gravity field to a sample of 838 Tully-Fisher galaxies; a maximum likelihood analysis leads to $\beta=$ $0.49 \pm 0.07$, which is consistent with our results for the IRAS gravity field. Similar low values of the density parameter emerge from the least action method applied to the flow field with $3000 \mathrm{~km} \mathrm{~s}^{-1}$ (Shaya et al. 1995). These procedures are distinct and do not all suffer from the same biases. Thus, it is encouraging that they are leading to consistent (and perhaps reliable) results.

In Figure 3, the individual peculiar velocities in the SN map appear to be slightly larger than the velocities predicted at the same locations by the IRAS or ORS fields. We attribute this to 
TABLE 1

Peculiar Velocity Data

\begin{tabular}{|c|c|c|c|c|c|c|c|}
\hline $\begin{array}{c}\text { SN Ia } \\
\text { (1) }\end{array}$ & $\begin{array}{c}l \\
(2)\end{array}$ & $\begin{array}{c}b \\
(3)\end{array}$ & $\begin{array}{c}c z \\
\left(\mathrm{~km} \mathrm{~s}^{-1}\right)\end{array}$ & $\begin{array}{c}c z-H_{0} d \\
\left(\mathrm{~km} \mathrm{~s}^{-1}\right) \\
(5)\end{array}$ & $\begin{array}{c}\sigma_{d} \\
\left(\mathrm{~km} \mathrm{~s}^{-1}\right) \\
(6)\end{array}$ & $\begin{array}{c}I R A S v \\
(\beta=0.4) \\
(7)\end{array}$ & $\begin{array}{c}\text { ORS } v \\
(\beta=0.3) \\
(8)\end{array}$ \\
\hline 1995al & 192.60 & 51.40 & 1493 & -466 & 123 & -407 & -365 \\
\hline 1996X & 310.20 & 35.70 & 1845 & -96 & 149 & -81 & -204 \\
\hline 1995D ....... & 230.00 & 39.67 & 2000 & -258 & 156 & -457 & -191 \\
\hline $1996 Z$ & 253.60 & 22.60 & 2014 & -427 & 289 & -320 & -71 \\
\hline $1991 \mathrm{M}$ & 30.39 & 45.90 & 2489 & 15 & 201 & -183 & -229 \\
\hline $1992 \mathrm{~K} \ldots \ldots$. & 306.28 & 16.31 & 2825 & -381 & 345 & -18 & 17 \\
\hline $1995 \mathrm{E} \ldots \ldots$ & 141.97 & 30.27 & 3639 & 175 & 217 & 21 & -368 \\
\hline 1991ag ....... & 342.56 & -31.64 & 4150 & -131 & 289 & 176 & 358 \\
\hline 1992al ........ & 347.30 & -38.50 & 4355 & 566 & 245 & 187 & 373 \\
\hline $1994 S \ldots . .$. & 187.84 & 85.75 & 4539 & 81 & 283 & -129 & -167 \\
\hline 1995bd ....... & 187.10 & -21.70 & 4808 & 227. & 329 & 46 & 193 \\
\hline 1993ae ...... & 144.62 & -63.23 & 5521 & 333 & 409 & 226 & 139 \\
\hline 1992bo ...... & 261.88 & -80.35 & 5662 & 424 & 369 & 99 & 327 \\
\hline $1992 b c \ldots .$. & 245.70 & -59.64 & 6053 & 551 & 355 & -59 & 282 \\
\hline 1994M ...... & 291.69 & 63.03 & 6730 & -716 & 564 & -407 & -29 \\
\hline 1995ak ...... & 169.70 & -49.00 & 6887 & 1063 & 578 & 252 & 221 \\
\hline $1993 \mathrm{H} \ldots . . .$. & 318.20 & 30.30 & 6982 & 263 & 443 & -156 & 62 \\
\hline 1992ag ....... & 312.50 & 38.40 & 7295 & -489 & 984 & -272 & 78 \\
\hline $1992 \mathrm{P} \ldots \ldots$ & 295.62 & 73.11 & 7447 & -519 & 539 & -408 & -108 \\
\hline 1994Q ....... & 99.60 & 65.00 & 8956 & -790 & 648 & -12 & -632 \\
\hline $1996 C \ldots \ldots$ & 64.38 & 39.68 & 8872 & -535 & 819 & 137 & -477 \\
\hline 1993ah ...... & 25.90 & -76.80 & 8974 & -53 & 1012 & 183 & 277 \\
\hline $19900 \ldots \ldots$ & 37.60 & 28.40 & 9247 & -1062 & 749 & 5 & -197 \\
\hline $1991 U \ldots \ldots$ & 311.82 & 36.21 & 9290 & 180 & 1357 & -260 & 23 \\
\hline
\end{tabular}

measurement noise and velocity noise (small-scale velocity flows) present in the $\mathrm{SN}$ data but not in the heavily smoothed IRAS or ORS velocity fields. Because of this noise, some SN velocity residuals do not match the sign (Fig. 3, filled symbols) of the IRAS predictions and/or the mode of the signs of the hemisphere in which they occur. But overall, the $\chi^{2}$ minimization for the SNs takes these sources of error properly into account and produces the best matched $\beta$ value for the data sets. Our determinations of $\beta$ are not sensitive to our estimate of the velocity noise or Type Ia SNs errors, the latter limited by the small dispersion from smooth Hubble flow. As the distances for individual SNs increase, the errors (in $\mathrm{km} \mathrm{s}^{-1}$ ) increase, while for the IRAS and ORS data sets the smoothing becomes stronger, and the amplitude of point-to-point variations gets smaller. Although more distant SNs carry less weight, we find that the objects within as well as beyond $5000 \mathrm{~km} \mathrm{~s}^{-1}$ give consistently low values for $\beta$. Nusser \& Davis (1995) show how to avoid the effects of mismatched smoothing that appear here, but the present SN sample is too sparse to apply their methods.
MLCS distances are precise enough to characterize the peculiar velocity field in the direction of each SN. Yet during this application we found that intrinsic uncertainties still limit the precision of relative Type Ia SN distances to no better than $5 \%$. Future investigations into observable SNs may improve the precision of these distances or our understanding of their limitations.

As the SN data set grows, the precision of this comparison between gravity and velocity will improve, and the methods of analysis that have been used on the galaxy data sets will become appropriate. No tool for mapping the peculiar velocity field has a brighter future.

This work was supported by NSF grant AST95-28340 and NASA grant NAG 5-1360 at UCB, NSF grants AST95-28899 and AST96-17058 at Harvard University, and by the Miller Institute for Basic Research in Science through a fellowship to A. G. R.

\section{REFERENCES}

Baker, J., Davis, M., Strauss, M., Lahav, O., \& Santiago, B. X. 1997, in preparation

da Costa, L., Freudling, W., Wegner, G., Giovanelli, R., Haynes, M., \& Salzer, J. 1996, ApJ, 468, L5

da Costa, L., Nusser, A., Freudling, W., Giovanelli, R., Haynes, M., Salzer, J., \& Wegner, G. 1997, astro-ph/9707299

Davis, M., Nusser, A., \& Willick, J. 1996, ApJ, 473, 22

Davis, M., \& Peebles, P. J. E. 1983, ApJ, 267, 465

Dekel, A. 1994, ARA\&A, 32, 371

Dekel, A., Bertschinger, E., \& Faber, S. M. 1990, ApJ, 364, 349

Dressler, A., Faber, S. M., Burstein, D., Davies, R. L., Lynden-Bell, D., Terlevich, R. J., \& Wegner, G. 1987, ApJ, 313, L37

Federspiel, M., Sandage, A., \& Tammann, G. A. 1994, ApJ, 430, 29

Fisher, K., Davis, M., Strauss, M. A., Yahil, A., \& Huchra, J. P. 1994, MNRAS, 266, 50

Fisher, K., Huchra, J. P., Strauss, M. A., Davis, M., Yahil, A., \& Schlegel, D. 1995, ApJS, 100, 69
Giovanelli, R., Haynes, M., Wegner, G., da Costa, L., Freudling, W., \& Salzer, J. 1996, ApJ, 464, L99

Hamuy, M., Phillips, M. M., Suntzeff, N. B., Schommer, R. A., Maza, R. A., \& Aviles, R. 1996, AJ, 112, 2048

Hamuy, M., et al. 1993, AJ, 106, 2392

Hudson, M. J. 1994, MNRAS, 266, 468

Jacoby, G., et al. 1992, PASP, 104, 599

Lauer, T., \& Postman, M. 1992, ApJ, 400, L47

- 1994, ApJ, 425, 418

Mathewson, D. S., \& Ford, V. L. 1994, ApJ, 434, L39

Nusser, A., \& Davis, M. 1994, ApJ, 421, L1 1995, MNRAS, 276, 1391

Peebles, P. J. E. 1980, The Large-Scale Structure of the Universe (Princeton: Princeton Univ. Press)

Press, W. H., Teukolsky, S. A., Vetterling, W. T., \& Flannery, B. P. 1992, Numerical Recipes (2d ed.; Cambridge: Cambridge Univ. Press)

Riess, A. G. 1996, Ph.D. thesis, Harvard Univ. 
Riess, A. G., Press W. H., \& Kirshner, R. P. 1995a, ApJ, 438, L17 1995b, ApJ, 445, L91

- 1996, ApJ, 473, 88

Riess, A. G., et al. 1997, in preparation

Santiago, B. X., et al. 1995, ApJ, 446, 457

Shaya, E., Peebles, P., \& Tully, B. 1995, ApJ, 454, 15

Sigad, Y., Dekel, A., Strauss, M., \& Yahil, A. 1997, in preparation
Strauss, M., \& Willick, J. 1995, Phys. Rep., 261, 271

Strauss, M. A., Yahil, A., Davis, M., Huchra, J. P., \& Fisher, K. B. 1992, ApJ, 397, 395

Tully, R. B., \& Fisher, J. R. 1977, A\&A, 54, 661

Willick, J. A., Courteau, S., Faber, S. M., Burstein, D., \& Dekel, A. 1995, ApJ, 446, 12

Willick, J., Strauss, M., Dekel, A., \& Kolatt, T. 1996, preprint 